\title{
Kaolinite/TiO $/$ /cobalt(II) Tetracarboxymetallophthalocyanine Nanocomposites as Heterogeneous Photocatalysts for Decomposition of Organic Pollutants Trimethoprim, Caffeine and Prometryn
}

\author{
Tiago H. da Silva, ${ }^{a}$ Anderson O. Ribeiro, ${ }^{b}$ Eduardo J. Nassar, ${ }^{\circledR a}$ Raquel Trujillano, ${ }^{c}$ \\ Vicente Rives, ${ }^{c}$ Miguel A. Vicente, ${ }^{c}$ Emerson H. de Faria ${ }^{*, a}$ and Katia J. Ciuffi ${ }^{\circledR *, a}$ \\ ${ }^{a}$ Universidade de Franca, Av. Dr. Armando Salles Oliveira, 201, \\ Pq. Universitário, 14404-600 Franca-SP, Brazil \\ ${ }^{b}$ Centro de Ciências Naturais e Humanas, Universidade Federal do ABC, \\ 09210-170 Santo André-SP, Brazil \\ 'GIR-QUESCAT, Departamento de Química Inorgánica, \\ Universidad de Salamanca, 37008 Salamanca, Spain
}

\begin{abstract}
São Simão's Brazilian kaolinite has been treated with titanium(IV) isopropoxide and cobalt(II) tetracarboxymetallophthalocyanine under different conditions (acidic or basic), leading, after drying at $100{ }^{\circ} \mathrm{C}$, to new titania-doped cobalt(II)-metallophthalocyanine/kaolinite solids. These solids were characterized by chemical analysis, powder X-ray diffraction, Fourier transform infrared spectroscopy, thermal analyses and nitrogen adsorption. No significant changes were observed by diffractometry, but the specific surface area depended of the synthetic route followed. The ability of these solids for photodegradation of trimethoprim, caffeine and prometryn was evaluated. The photodegradation was followed via mass spectrometry and UV-Vis absorption spectroscopy. The presence of photodegradation by-products was verified in all cases. All the photocatalysts showed high photodegradation rate against prometryn, trimethoprim and caffeine, the degradation efficiencies were 54, 30 and 90\%, respectively, when using the heterogeneous photocatalysts. Comparison with commercial $\mathrm{TiO}_{2}\left(\right.$ Degusa $\left.{ }^{\circledR}\right)$ proved that the synthesized photocatalyst based on kaolinite present higher degradation rate than isolated titanium dioxide.
\end{abstract}

Keywords: clay minerals, sol-gel, heterogeneous photocatalysis

\section{Introduction}

The humankind uses large amounts of chemicals/ pharmaceuticals in numerous areas as cosmetics, medicine and industrial. ${ }^{1,2}$ This excessive consumption could promote an exponential increase in the productive processes of these compounds, which results on the discharge of untreated wastes or partially treated wastewater in natural environments. This incorrect disposal may directly contaminate surface or underground water. ${ }^{3}$ In this way, the concern about drinking water should lead to the development of more efficient processes for the treatment of water and wastewater, aiming at environmental preservation. In this context, advanced oxidation process is a promising technology that does not require large cost for

*e-mail: eh.defaria@gmail.com; katia.ciuffi@unifran.edu.br up-scaling to technological application and results in higher yields of degradation of organic compounds promoting in some cases the complete mineralization of the pollutants.

Nowadays, the emerging organic pollutants (EOPs) are defined as synthetic or naturally occurring chemicals that are not commonly monitored in the environment, but which have the potential to enter the environment and cause known or suspected adverse ecological and (or) human health effects. More than 700 emerging pollutants, their metabolites and transformation products, have been found in European aquatic environments. ${ }^{4}$ These emerging pollutants, and their metabolites and transformation products, have been listed by Norman Network (2017). ${ }^{5}$

In this context, trimethoprim [2,4-diamine 5-(3,4,5-trimethoxybenzyl)pyrimidine] (TMP) is a widely used antibacterial drug for treatment of urinary, respiratory or gastrointestinal infections, with application in humans 
and animals. ${ }^{6,7}$ However, TMP is incompletely metabolized by humans during the therapeutic process and about $80 \%$ is excreted in the pharmacologically active form, which can promote the development of bacterial resistance to this medicine, ${ }^{7-10}$ converting this compound in an EOP.

Another example of EOP is caffeine (1,3,7-trimethylxanthine) (CFF), which belongs to the family of methylxanthines and can be classified as an alkaloid; it is consumed regularly around the world, e.g., beverages, pharmaceuticals and products for personal care, becoming a chemical marker of the pollution of surface water and environmental pollution indicator. Caffeine features characteristic stimulant, psychoactive properties, acting on the central nervous system of humans, promoting changes in sleep and agility. ${ }^{11,12}$ Large amounts of caffeine occur in the seeds, leaves, and fruits of some plants, the microorganisms existing in the environment cannot metabolize this compound satisfactorily.

Another important pollutant is prometryn (2,4-bis(isopropylamino)-6-(methylthio)-1,3,5-triazine) (PMT), a triazine-class herbicide widely used to control grass and weeds in a range of agro-industrial crops. This class of herbicides, due to their chemical stability, settles in the soil for a large period of time and consequently contaminates water bodies, as groundwater. ${ }^{13,14}$ Prometryn is considered a moderately persistent chemical and listed in European Commission Health and Food Safety guidelines as toxic compound, ${ }^{13-15}$ needing the development of methods for its control and remediation.

Considering the great problem of environmental contamination by EOPs, the advanced oxidation processes (AOPs) show a great potential for their elimination. That is, heterogeneous photocatalysis using semiconducting materials emerges as a promising strategy for the protection of the ecosystem and human health because it is effective in the treatment of pharmaceuticals, dyes, herbicides and pesticides, in different concentration ranges, unlike conventional biological treatment processes, which have difficulties for their complete removal. , $3,16-20^{2}$

Some materials stand out in photocatalytic processes for environmental remediation, while particles of titanium dioxide $\left(\mathrm{TiO}_{2}\right)$ stand out as the most used, functional, versatile and effective photocatalyst, due to its photoelectric properties, low cost, corrosion resistance and non-toxicity. ${ }^{21-23}$ One of the problems for using of $\mathrm{TiO}_{2}$ is its low energy efficiency, its energy band gap is $3.2 \mathrm{eV}$, equivalent to the ultraviolet wavelength of $387.5 \mathrm{~nm}$, which corresponds to only 3 to $5 \%$ of the solar radiation reaching the Earth surface. ${ }^{3,24,25}$ This problem can be mitigated with some procedures, such as the dispersion on inorganic matrices, ${ }^{17,26,27}$ such as clay minerals. Clay minerals are attractive materials for many significant applications, due to their physical and chemical properties, including catalysis/photocatalysis and adsorption, ${ }^{12,28,29}$ especially kaolinite, whose layered structure provides the possibility of retention of a great variety of compounds to reduce the energy required for the activation of the semiconductor. Catalyst improvement via immobilization might also be due to the force field between the support and the $\mathrm{TiO}_{2}$ particles that inhibits the recombination of electron-hole pairs. ${ }^{17}$

The metallophtalocyanines molecules consist of aromatic symmetrical macrocycles with benzopyrole rings connected by nitrogen links that provide a $\pi$ electron arrangement, providing to the metallophtalocyanines a high absorption coefficient in the ultraviolet/visible region, similar to that of porphyrins, favoring the transfer of energy to the semiconductor. ${ }^{30-32}$

Considering the possibility of improving the catalytic properties of $\mathrm{TiO}_{2}$ associated to its low energy conversion by dispersion on inorganic matrices along with complexation of organometallic compounds such as metallophtalocyanines, we here report on the preparation of kaolinite/ $/ \mathrm{TiO}_{2} /$ metallophtalocyanines composites, and their application for the degradation of various pollutants, namely, trimethoprim, caffeine and prometryn (Table S1, Supplementary Information (SI) section).

\section{Experimental}

\section{Purification of kaolinite}

The kaolinite used in this work was provided by the mining company Darcy R.O. Silva \& Cia, located in the city of São Simão (Brazil). The raw clay mineral was purified by the dispersion-decantation method, ${ }^{33,34}$ and the purified kaolinite was designated Kaol.

\section{Synthesis of the photocatalysts}

The photocatalyst materials were obtained by the sol-gel method, as described by Barbosa et al. ${ }^{17} \mathrm{~A}$ mixture of ethanol (200 mL), kaolinite (38.02 mmol), titanium(IV) isopropoxide ( $\left.\mathrm{Ti}^{\mathrm{IV}} \mathrm{IP}\right)(8.444 \mathrm{mmol})$ and cobalt(II) tetracarboxyphthalocyanine (CoMPc) (0.1939 mmol) was submitted to strong magnetic stirring for $24 \mathrm{~h}$ at room temperature, in acidic (acetic acid-17.49 $\mathrm{mmol}$ ) or basic (sodium hydroxide-15 mmol) medium, to study the influence of $\mathrm{pH}$ on the formation of the material. The hydrolysis/condensation process is schematized in Figure S1 (SI section).

The samples were denoted as CAR (composite acid route), that is, $\mathrm{Kaol}-\mathrm{Ti}-\mathrm{H}^{+}$, a reference solid obtained 
carrying out the entire process but without the addition of CoMPc, and CAR-CoMPc with the addition of the organometallic compound. Analogously, for the synthesis by the basic route, the solids obtained were denoted CBR (composite basic route, without CoMPc) and CBR-CoMPc with the addition of the metallophtalocyanine, respectively. All the materials were submitted to heat treatment $\left(400^{\circ} \mathrm{C}\right)$ for $3 \mathrm{~h}$ for the removal of solvents and stabilization of the $\mathrm{TiO}_{2}$ phase, and washed to remove possible non-attached oxides formed during the thermal treatment.

\section{Photocatalysis study}

For the photodegradation reaction studies, a MPDSBasic system from Peschl Ultraviolet, with a PhotoLAB Batch-L reactor and a TQ150-Z0 lamp (power $150 \mathrm{~W}$ ), integrated in a photon CABINET, was used. The spectrum is continuous, with the main peaks at $546 \mathrm{~nm}$ (radiation flux $(\varphi) 5.1 \mathrm{~W}), 366 \mathrm{~nm}(\varphi 6.4 \mathrm{~W}), 313 \mathrm{~nm}(\varphi 4.3 \mathrm{~W})$ and $254 \mathrm{~nm}(\varphi 4 \mathrm{~W})$. The reactor is vertically oriented and it is refrigerated by circulating cold water. In each reaction, $750 \mathrm{mg}$ of catalyst was added to a contaminant solution of $25 \mathrm{mg} \mathrm{L}^{-1}$ (TMP and CFF) in $\mathrm{H}_{2} \mathrm{O}$ and $10 \mathrm{mg} \mathrm{L}^{-1}$ of PMT in a $1: 1$ ethanol: $\mathrm{H}_{2} \mathrm{O}$ mixture, due to the low solubility in water, although the presence of ethanol is not expected to interfere in the catalytic reaction. The concentration of the contaminants was determined by UV-Vis spectroscopy at pre-determined time intervals between 15 and $240 \mathrm{~min}$, using a PerkinElmer Lambda 35 spectrophotometer. To identify the by-products generated during UV degradation, the solutions were analyzed by mass spectrometry after various treatment times. The equipment used for this purpose was an Agilent 1100 HPLC apparatus coupled to an ultraviolet detector and an Agilent Trap XCT mass spectrometer. These analyses were carried out at Servicio Central de Análisis Elemental, Cromatografía y Masas (Universidad de Salamanca).

\section{Evaluation of photodegradation mechanism}

In a typical experiment, a $10 \mathrm{mg} \mathrm{L}^{-1}$ aqueous solution of PMT was continuously stirred magnetically through the reactor. A $5 \mathrm{~mL}$ aliquot was collected and analyzed by UV-Visible spectroscopy (UV-Vis). The effect of chloride ions on the photocatalytic activity was investigated by adding sodium chloride $(\mathrm{NaCl})$ (concentration $16.6 \mathrm{mg} \mathrm{L}^{-1}$ ) to the initial PMT solution, using the described system.

\section{Characterization techniques}

Chemical analyses were carried out by using a mass spectrometer with inductive coupling plasma source (ICP-MS) Elan 6000 from PerkinElmer Sciex equipped with an autosampler located in a laminar flow hood. The powder X-ray diffraction (PXRD) diagrams of the solids were recorded in a Siemens D-5000 diffractometer operating at $40 \mathrm{kV}$ and $30 \mathrm{~mA}$, using filtered $\mathrm{Cu} \mathrm{K} \alpha$ radiation in the $2-65^{\circ}(2 \theta)$ range. All the analyses were carried out at a scan speed of $2^{\circ} \mathrm{min}^{-1}$. The thermal analyses were carried out in a TA Instruments SDTQ600 simultaneous DTA-TGA thermal analyzer, at temperatures ranging from 25 to $900{ }^{\circ} \mathrm{C}$, at a heating rate of $10^{\circ} \mathrm{C} \mathrm{min}^{-1}$ and under air flow $\left(100 \mathrm{~mL} \mathrm{~min}^{-1}\right)$. The infrared absorption spectra were recorded in a PerkinElmer FTIR Frontier Spectrometer by using a diffuse reflectance accessory, using the $\mathrm{KBr}$ pellet technique. The Brunauer-Emmett-Teller (BET) specific surface area and porosity data of the solids were calculated from their nitrogen adsorption-desorption isotherms at $-196^{\circ} \mathrm{C}$, recorded in a Micromeritics Gemini VII $2390 \mathrm{~T}$ apparatus. The samples were previously treated at $110^{\circ} \mathrm{C}$ under a stream of $\mathrm{N}_{2}$ for $2 \mathrm{~h}$, in a Micromeritics Flowprep 060 equipment. The absorption spectra in the UV-Visible region were obtained in a PerkinElmer Lambda 35 spectrophotometer, coupled to a computer with UV WinLab 2.85 software. Calibration curves were used for the pollutants, verifying that they followed the LambertBeer law in the concentration range used in the work. The wavelengths of maximum absorbance were $286 \mathrm{~nm}$ for TMP, $273 \mathrm{~nm}$ for CFF and $223 \mathrm{~nm}$ for PMT, respectively.

\section{Results and Discussion}

\section{Characterization of the solids}

The thermogravimetric (TG) curves of the two composites containing the metallophtalocyanines (Figure 1) were very similar to those from parent kaolinite and for the composites without metallophtalocyanines (Figure S2, SI section). Both CAR-CoMPc and CBR-CoMPc materials showed mass losses related to the elimination of water and solvent close to $60{ }^{\circ} \mathrm{C}$. From this temperature up to ca. $400^{\circ} \mathrm{C}$, removal of organic matter from the precursors was observed as a gently process, followed by the strong mass loss due to dehydroxylation of kaolinite. The dehydroxylation temperature and mass percentage were different from those in parent kaolinite. Thus, for sample CAR-CoMPc the mass loss between $400-900{ }^{\circ} \mathrm{C}$ was $13.44 \%$, suggesting the presence of organic matter and/or hydroxyl groups derived from the formation of the polymeric titanium network and immobilized CoMPc, and probably related to the high thermal stability of metallophtalocyanines. ${ }^{32}$ For the CBR-CoMPc solid, this mass loss was $11.69 \%$, suggesting the presence of a lower 
concentration of hydroxyl groups and CoMPc, probably because of the formation of larger titania aggregates, hindering the immobilization of CoMPc. The thermal curves of composites without CoMPc were very similar, only noting small differences in the mass loss percentages, attributable to differences in the amount of organic matter and of hydroxyl groups. It was also observed that despite very similar amounts of sample were used in the thermal studies, the intensity of the differential thermal analysis (DTA) signals was much stronger for sample CAR-CoMPc than for sample CBR-CoMPc, although the mass losses (percentage) were similar in both cases. Due to the low $\mathrm{pH}$ value, the carboxylic group was not deprotonated in the acid route (sample CAR-CoMPc) and could react with the titania-kaolinite substrate forming a covalent bond; however, deprotonated carboxylate groups could be coordinated to coordinatively unsaturated (cus) $\mathrm{Ti}^{4+}$ cations at the titania surface.

Chemical composition of kaolinite (Table 1) was very similar to that previously reported, ${ }^{33}$ with small differences attributable to isomorphous substitutions. The $\mathrm{Si} / \mathrm{Al}$ ratio is an important information for kaolinite-derived materials; this ratio is hardly changed, as it would require extreme chemical reactions. The ratio for the parent sample was

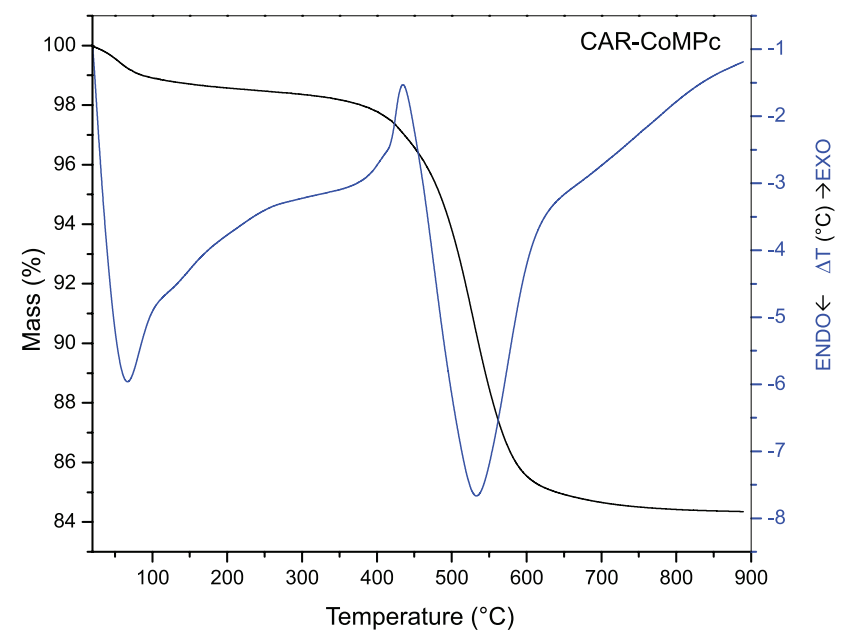

1.028. For samples CAR and CBR, this ratio was 1.020 and 1.023 , respectively, indicating that the structure of kaolinite was not affected by the incorporation of Ti-species, while for samples CAR-CoMPc and CBR-CoMPc this ratio decreased to 0.999 and 0.962 , respectively, suggesting the solubilization of a small amount of silica from the tetrahedral sheet of kaolinite, although a priori it was not expectable that the presence of the metallophtalocyanine may cause this process. ${ }^{17,32}$

The content of $\mathrm{TiO}_{2}$ strongly changed from parent kaolinite to all the composites. The amount of supported $\mathrm{TiO}_{2}$ was moderate in the composites prepared in the absence of CoMPc (0.6-1.1\%), and much higher, 5.7-6.5\%, in the presence of this compound, which suggested that the presence of CoMPc strongly affected the hydrolysis of the Ti-precursor; in fact, the Ti precursor can hydrolyze on carboxylate group of CoMPc, as proposed in Figure S1 (SI section). The presence of so different titania contents could be related to the relative importance of the hydrolysis of the titanium precursor and the condensation steps during the formation of the hybrid materials. Hydrolysis was favored in the acid route ( $\mathrm{pH}$ close to 4), leading to the formation of a large concentration of Ti-OH units and thus a larger number of small titania clusters would be

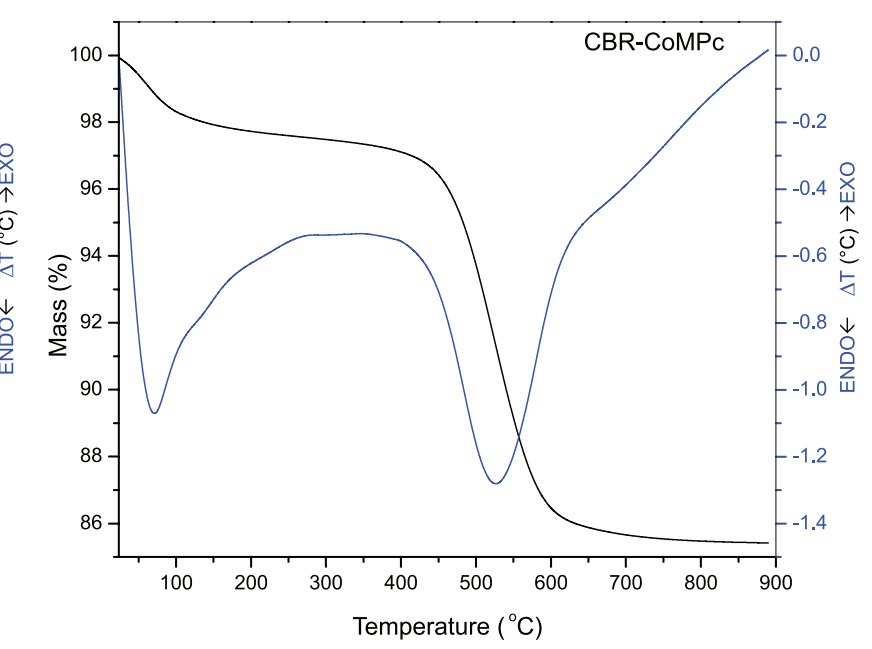

Figure 1. TG/DTA curves of materials derived from kaolinite.

Table 1. Chemical composition of the materials, expressed in the free-water form

\begin{tabular}{lccccccccc}
\hline & $\mathrm{SiO}_{2}$ & $\mathrm{Al}_{2} \mathrm{O}_{3}$ & $\mathrm{Fe}_{2} \mathrm{O}_{3}$ & $\mathrm{MnO}$ & $\mathrm{MgO}$ & $\mathrm{CaO}$ & $\mathrm{Na}_{2} \mathrm{O}$ & $\mathrm{K}_{2} \mathrm{O}$ & $\mathrm{TiO}_{2}$ \\
\hline Kaol & 53.02 & 43.84 & 1.12 & 0.01 & 0.27 & 0.09 & 0.06 & 0.44 & 1.14 \\
CAR & 52.31 & 43.59 & 1.09 & 0.01 & 0.27 & 0.08 & 0.02 & 0.44 & 2.19 \\
CBR & 48.60 & 40.39 & 1.06 & 0.01 & 0.26 & 0.08 & 1.57 & 0.42 & 7.60 \\
CAR-CoMPc & 52.00 & 44.27 & 1.11 & 0.01 & 0.27 & 0.08 & 0.02 & 0.43 & 1.80 \\
CBR-CoMPc & 48.06 & 42.47 & 0.99 & 0.01 & 0.24 & 0.08 & 0.87 & 0.42 & 6.86 \\
\hline
\end{tabular}

CAR: composite acid route; CBR: composite basic route; CoMPc: cobalt(II) tetracarboxymetallophthalocyanine. 
formed. The basic route ( $\mathrm{pH}$ close to 12) would give rise to larger precipitation of titania particles, which agglomerate forming large particles.

The acid route promoted the protonation of the alkoxide groups, and preferentially directed the crystalline network to the edges of the kaolinite structure, resulting in a more extended and less branched inorganic polymeric network. Through the basic route, the alkoxide deprotonated, promoting the decrease of the hydrolysis rate, but at the same time improving the condensation kinetics, forming more agglomerated and highly branched species. These facts could explain why the amount of titanium oxide deposited on sample CAR was smaller than on sample CBR. ${ }^{35}$

According to the X-ray diffractograms, the reaction of kaolinite with Ti(IV)IP and CoMPc did not promote any change in the structure of the former (Figure 2). Thus, basal spacing of kaolinite remained close to $7.15 \AA$ in all the solids. Kaolinite is usually submitted to a previous swelling treatment with highly polar molecules, e.g., dimethylsulfoxide, to favor intercalation of other species. However, this treatment was not applied in the present case, so it was expectable that the reactions taking place with the other reactants would take place only on the kaolinite surface and on the edges of the crystals and not in the interlayer space, so without swelling of the kaolinite structure.

On other hand, no reflections from Ti-containing species were observed. However, the decrease in the relative intensity of the basal reflection suggested some sort of interaction between kaolinite, $\mathrm{CoMPc}$ and $\mathrm{TiO}_{2}$ (Figure 2). This decrease may be caused by the covering of kaolinite surface with titanium alkoxide species, and the attachment of titanium dioxide and CoMPc to the kaolinite layers after hydrolysis and condensation of the alkoxide, interacting with the kaolinite surface. ${ }^{36-39}$

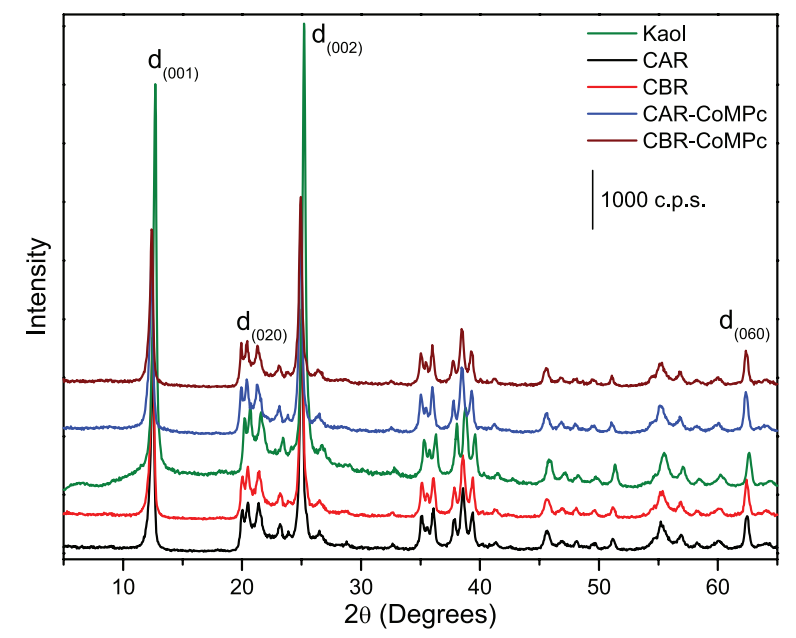

Figure 2. Diffractograms of the kaolinite-derived samples.
The intensities of reflections (001) and (002) and also the ratio between their intensities-(001)/(020)-decreased for the derived materials (CAR, CBR, CAR-CoMPc and CBR-CoMPc) (Table S2, SI section), suggesting that the treatments could promote changes in the stacking of the kaolinite layers. Other evidence was the decrease in the ratio between the intensities of the reflections (020) and (006). This last reflection is characteristic of the $a b$ plane; the absence of changes in directions $a b$ suggested that this decrease was related to changes in the stacking of plates along the $c$-direction, with a lower number of neatly stacked layered structures. ${ }^{36-39}$ These results also demonstrated that the acidic or basic conditions used in the preparation of the samples were not aggressive enough to cause changes in the structure of kaolinite. No peaks due to $\mathrm{TiO}_{2}$ phases were recorded, although it may be remarked that the characteristic reflections of anatase are very close to those from kaolinite, a fact which made difficult the analysis on these solids.

Based on Fourier transform infrared (FTIR) spectra (Figure S3, SI section), the synthesis of photocatalysts containing $\mathrm{TiO}_{2}$ and $\mathrm{CoMPc}$ did not promote any changes on kaolinite interlayer region, the typical interlayer hydroxyl bands at 3696, 3670, $3654 \mathrm{~cm}^{-1}$ (inner surface) and $3621 \mathrm{~cm}^{-1}$ (inner) remain intact after reactions in acidic or alkaline media. ${ }^{40,41}$ The presence of CoMPc on solid $\mathrm{Kaol} / \mathrm{TiO}_{2}$ was evidenced by the presence of vibrations at 1400 and $1415 \mathrm{~cm}^{-1}$ assigned to $\mathrm{C}=\mathrm{C}$ units from the aromatic ring, and bands at 1332, 1335 and $1563 \mathrm{~cm}^{-1}$ assigned to $\mathrm{C}-\mathrm{N}$ stretching modes of aromatic amines from the conjugated macrocyclic ring. ${ }^{40}$

The nitrogen adsorption-desorption isotherms recorded for the solids (Figure S4, SI section) were classified as type III. ${ }^{17,42}$ Their similarities confirmed that the texture of the inorganic matrix did not change drastically with the treatments applied. Deposition of $\mathrm{TiO}_{2}$ and CoMPc on the surface of kaolinite led to small increases in the specific surface area (SSA) values, while porosity remained practically constant (Table 2). The solids containing metallophtalocyanine showed slightly higher values than the solids without it, which suggested that the presence of CoMPc in the reaction medium could lead to more disperse Ti-species, by the development of sites able to promote the adsorption. ${ }^{17,32}$

The band gap of the different solids was estimated from their absorption spectra (Figure S5, SI section), by means of the Tauc plot approximation. ${ }^{43}$ This method allowed to determine the band edge by applying the equation 1 :

$\alpha h v=A\left(h v-E_{g}\right)^{1 / 2}$

where $\alpha, \mathrm{h}, \nu, \mathrm{E}_{\mathrm{g}}$ and A denote the adsorption coefficient, 
Planck constant, radiation frequency, band gap and a constant, respectively. From this equation, a plot of $(\alpha h v)^{1 / 2} v s . h v$, the so-called Tauc plot, showed a linear region just above the absorption edge whose extrapolation to the photon energy axis (hv) provides the semiconductor band gap value. The presence of CoMPc strongly influenced the band gap, which may induce changes in the absorption ability of the solids and thereof on their photocatalytic properties (Table 2). The solids containing CoMPc exhibited UV-Vis absorption bands at 550-800 nm (Figure S5, SI section). These two absorption bands were assigned to $\mathrm{Q}$ bands of CoMPc from $\pi-\pi^{*}$ transitions which confirmed that the solids containing CoMPc promoted the shift and broadness of the bands to the visible region. Thus, the materials containing CoMPc may inject the electrons to the conduction band $(\mathrm{CB})$ of $\mathrm{TiO}_{2}$ deposited on kaolinite surfaces, allowing $\mathrm{CB}$ to act as electron mediator to transfer them from CoMPc to electron acceptors on $\mathrm{TiO}_{2}$ surfaces, maintaining intact the valence band (VB). ${ }^{44}$

Table 2. Textural data and band gap of the materials

\begin{tabular}{lccc}
\hline & $\mathrm{S}_{\mathrm{BET}} /\left(\mathrm{m}^{2} \mathrm{~g}^{-1}\right)$ & $\mathrm{V}_{\mathrm{P}} /\left(\mathrm{cm}^{3} \mathrm{~g}^{-1}\right)$ & Band gap / eV \\
\hline Kaol & 23 & 0.061 & 4.79 \\
CAR & 26 & 0.063 & 4.65 \\
CBR & 23 & 0.058 & 4.75 \\
CAR-CoMPc & 29 & 0.067 & 1.82 \\
CBR-CoMPc & 34 & 0.065 & 1.78
\end{tabular}

$\mathrm{S}_{\mathrm{BET}}$ : BET specific surface area; $\mathrm{V}_{\mathrm{p}}$ : total pore volume (at $\mathrm{p} / \mathrm{p}^{0}=0.99$ ); CAR: composite acid route; CBR: composite basic route; CoMPc: cobalt(II) tetracarboxymetallophthalocyanine.

Immobilization of $\mathrm{TiO}_{2}$ in kaolinite modified only slightly its band gap, probably because of the high purity of kaolinite, which did not present any group that could absorb energy for possible transference, and by the low amount of $\mathrm{TiO}_{2}$ incorporated in these materials, lower than $8 \% \mathrm{TiO}_{2}$ in both cases. However, samples CAR-CoMPc and CBR-CoMPc showed a drastic band gap decrease, assigned to the presence of CoMPc, which may induce the antenna effect interacting with the $\mathrm{TiO}_{2}$ existing on the kaolinite surface, absorbing energy and transferring it to $\mathrm{TiO}_{2}$. This is a similar effect to that reported by González et al..$^{45}$ when using different cations as doping agents in Ti-pillared montmorillonite.

\section{Photocatalytic degradation of pollutants}

Removal of the pollutants TMP, CFF and PMT was estimated by UV-Vis spectroscopy. In addition, the by-products formed were identified by mass spectrometry
(MS), also aiming to gain information on the degradation route under the reaction conditions used.

Photolysis of TMP was very significant, $72 \%$ after 240 min (Figure 3), observed by the decrease in intensity/ disappearance of the corresponding signals both in UV-Vis and MS. ${ }^{9}$ The typical molecular ion signal with a mass/charge ratio $(\mathrm{m} / \mathrm{z})$ of 291.3 disappeared, indicating that all TMP underwent photodegradation, but not complete mineralization, as fragments with $\mathrm{m} / \mathrm{z}$ and 243 and 111 were recorded (Figure S6, SI section), indicating the formation of by-products/intermediates. These fragments may absorb close to the wavelength used for evaluating TMP (mainly the low degraded fragment with $\mathrm{m} / \mathrm{z} 243$ ), thus leading to false results from UV-Vis. In other words, the remaining TMP amount found may in fact be due to byproducts generated during the photodegradation process.

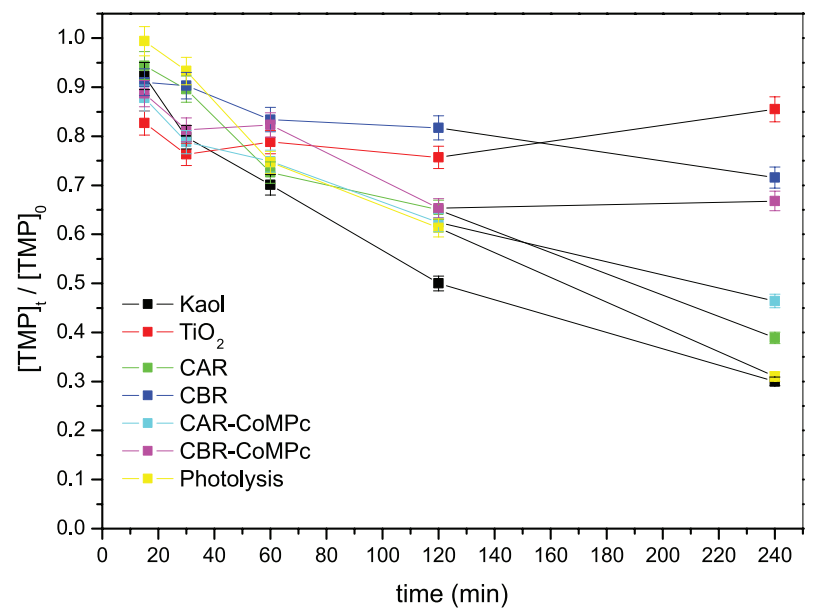

Figure 3. Kinetic behavior $\left(\mathrm{C}_{\mathrm{t}} / \mathrm{C}_{0}\right)$ of the photodegradation of trimethoprim.

Parent kaolinite showed a good efficiency in the photodegradation of TMP, probably due to the presence of active metal species in its structure, $\mathrm{Fe}^{3+}$ and $\mathrm{Ti}^{4+}$. It may be remarked that kaolinite adsorbed a small amount of TMP, which in fact may cause the decrease in the intensity of the TMP absorption band in the UV-Vis spectrum.

The composites showed a better photocatalytic behavior than Kaol at short reaction times; however, after $60 \mathrm{~min}$ of reaction all the materials showed similar efficiencies, evidencing that a very complex process was involved in TMP photodegradation. This may be limited by the very low amount of $\mathrm{TiO}_{2}$ in the composites, but also by a blocking effect promoted by CoMPc, hindering the generation of $\mathrm{OH} \bullet$ radicals associated to $\mathrm{TiO}_{2}$ on kaolinite. This effect was actually confirmed, as the amount of TMP photodegraded was higher for the solids without CoMPc than for the solids containing it. This finding strongly suggested that although CoMPc increased the range of 
light absorption, at the same time CoMPc may difficult the access to active sites from $\mathrm{TiO}_{2}$. Other possibilities were that the absorbed energy was transferred to $\mathrm{Co}^{2+}$ or to the reaction medium, this last hypothesis was supported by the fact that the final temperature after the reaction was $40-45^{\circ}$ (vs. $25^{\circ} \mathrm{C}$ at the beginning of the reaction). Even CoMPc itself may be photodegraded, as suggested by the color change in the catalysts, blue at the beginning of the reaction and almost white at the end.

For the CBR-CoMPc solid, and also for commercial P25 $\mathrm{TiO}_{2}$, used as reference photocatalyst, the concentration of TMP did not follow the decrease with time pattern observed in photolysis process and for the other catalysts. In these two cases, the concentration of TMP after 240 min seemed to be greater than after $120 \mathrm{~min}$. This strongly suggested the formation of intermediates that absorbed in the same wavelength region than TMP, and/or of fragments that may slowly recombine in solution. This resulted in complex photodegradation patterns, consequently causing a decrease in the efficiency of generation of hydroxyl $(\mathrm{OH} \bullet)$ in the reaction medium.

It is remarkable that the content of $\mathrm{TiO}_{2}$ in the composites was much lower (between 1.80 and $7.60 \%$ ) than the values usually reported in the literature, where contents above $50 \%$ or even $90 \% \mathrm{TiO}_{2}$ on inorganic matrices have been used. ${ }^{22,26}$ Thus, the performance of our materials was similar to materials with much higher $\mathrm{TiO}_{2}$ content, being remarkable the fast degradation rate observed at short times of reaction. It may be also considered that no oxidant agent was added to improve the degradation.

$\mathrm{Cai}$ and $\mathrm{Hu}^{46}$ reported the degradation of sulfamethoxazole and trimethoprim using the photocatalytic system formed by $\mathrm{TiO}_{2}$ and UV-A radiation produced by light-emitting diodes (UVA(LED)/ $/ \mathrm{TiO}_{2}$ ), adding $\mathrm{H}_{2} \mathrm{O}_{2}$ as an oxidizing agent. The system showed satisfactory removal of the antibiotics, and the efficiency was directly linked to the reaction conditions. Approximately $90 \%$ of the initial concentration of antibiotics was degraded after $20 \mathrm{~min}$ of reaction, forming intermediates which precise nature also depended of the reaction conditions. The initial steps of the degradation should involve the hydroxylation and cleavage of $-\mathrm{NH}-$ bonds, resulting in the formation of benzene, aniline and phenol. In fact, these byproducts were evidenced by UV-Vis spectroscopy and confirmed by mass spectrometry. Some signals were found in the MS spectra (Figure S6, SI section), their $m / z$ ratios agreed with those reported by Ji et al., ${ }^{9}$ thus allowing to propose the degradation route included in Figure 4. According to this route, fragments with high $\mathrm{m} / \mathrm{z}$ values were formed, which essentially maintained the structure of the molecule (except the fragment with $\mathrm{m} / \mathrm{z}$ 111). It was evident that complete mineralization was not achieved under these reaction conditions, but breaking the molecule through different positions may probably provide further degradation.

Photodegradation of PMT was also difficult, reaching maximum values close to $40 \%$. Before the degradation

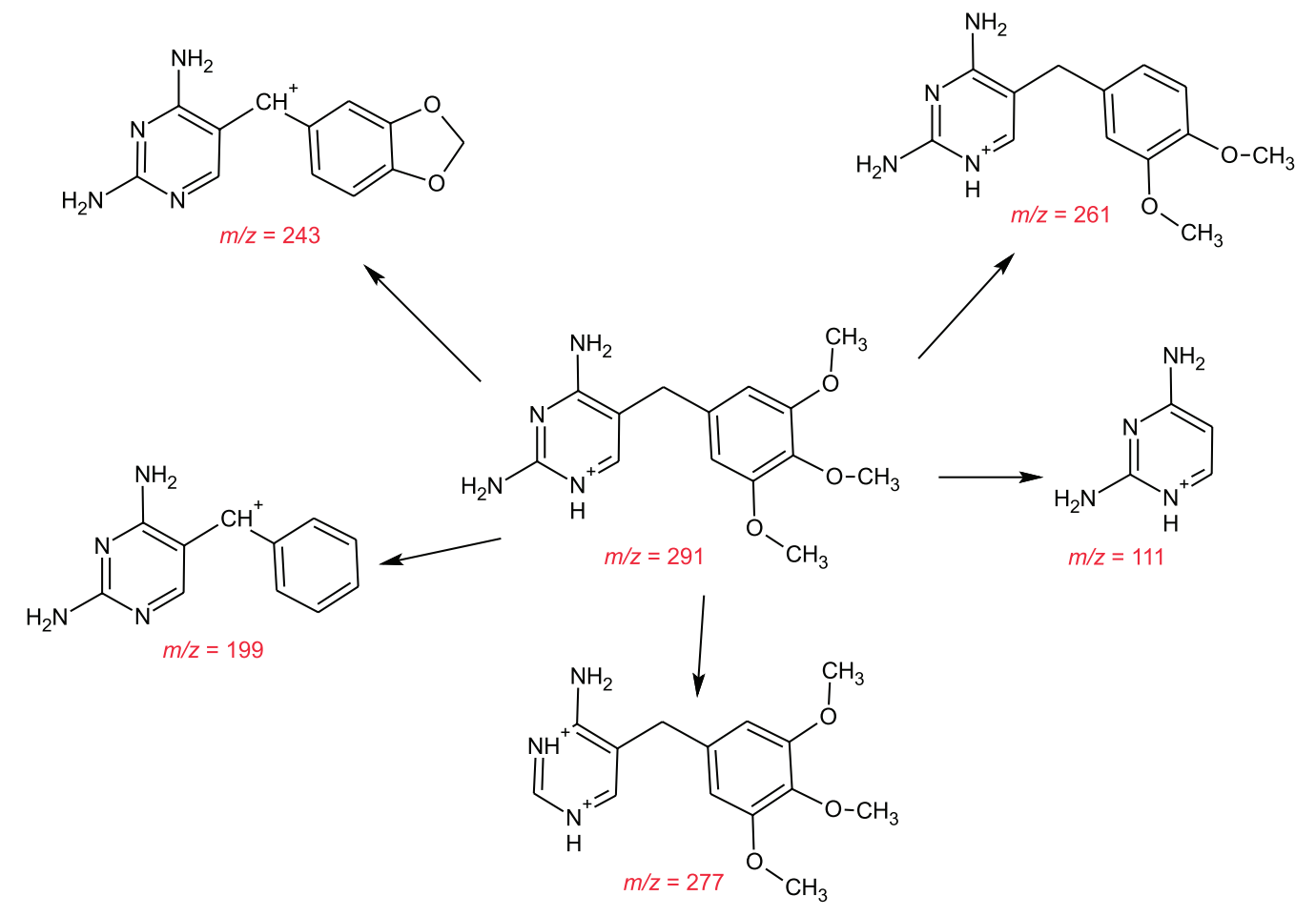

Figure 4. Proposal for the photocatalytic decomposition of TMP. 
study, adsorption tests were carried out (denoted as "Dark experiments" in Figure 5); the adsorption was very low for all the solids. In the degradation study, the best results were again found for photolysis and Kaol. In the case of Kaol, the activity could be explained as above for TMP degradation, by the presence of active cations $\mathrm{Fe}^{3+}$ and $\mathrm{Ti}^{4+}$. For the four composites, without and with metallophthalocyanine, the degradation efficiency decreased; a finding again attributable to the difficult access to the catalytically active sites or to the formation of stable by-products that may absorb in the same region than TMP in the UV-Vis spectroscopy studies. Although the differences were not very large, the materials obtained through the acid route showed better results, attributable to a better access to the active sites.

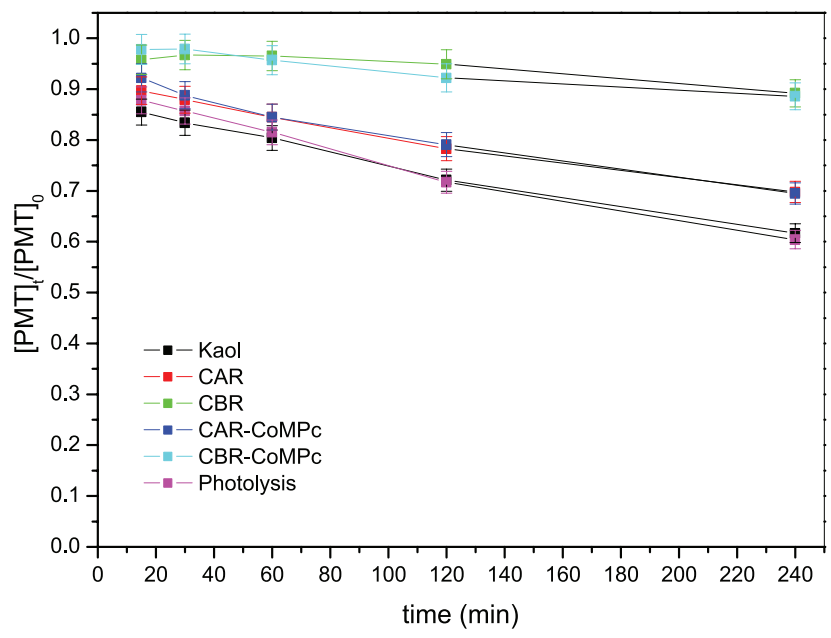

The MS spectra (Figure S7, SI section) showed the formation of various by-products after the photocatalytic process, all of them with high $\mathrm{m} / \mathrm{z}$ values, suggesting that the central ring of the molecule remained unchanged. The fragment with $\mathrm{m} / \mathrm{z}, 196$ may be particularly stable, as this signal appeared for all materials studied and also in the photolysis runs. The possible structure of the fragments detected by MS is given in Figure 6. ${ }^{14,47}$ As indicated, all fragments maintained the chromophoric triazine ring structure, which confirmed the difficulty to quantify the photodegradation of PMT by UV-Vis, because these fragments may absorb very close to the positions of the bands due to the PMT molecule.

Evgenidou et al. ${ }^{15}$ reported PMT degradation using two

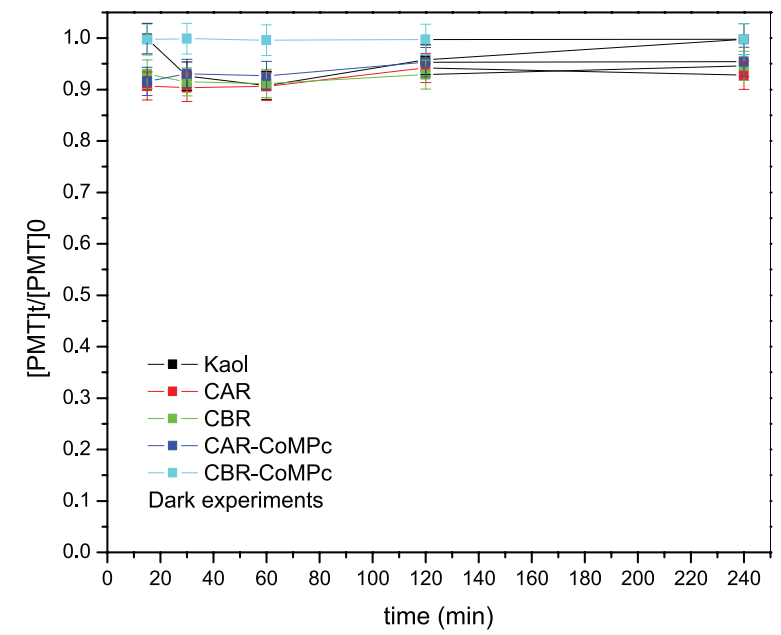

Figure 5. Kinetic behavior $\left(C_{1} / C_{0}\right)$ of the photodegradation of prometryn.<smiles></smiles>

Figure 6. Proposal for the photocatalytic decomposition of PMT. 
types of $\mathrm{TiO}_{2}$ as photocatalysts, demonstrating that Degussa P-25 was more effective than UV-100 Hombikat. Using $\mathrm{H}_{2} \mathrm{O}_{2}$ and $\mathrm{K}_{2} \mathrm{~S}_{2} \mathrm{O}_{8}$ as oxidizing agents, percentages around $70 \%$ were reached. These authors claimed the formation of by-products and the difficulty for the complete degradation of the triazine ring.

Caffeine was much more sensitive to degradation than trimethoprim and prometryn, reaching values between 85-95\% (Figure 7). Adsorption experiments were also carried out before the photodegradation studies (Figure 7, "Dark experiments"), finding that adsorption was low, in some cases almost null and always lower than $10 \%$. Although at the end of the reaction $(240 \mathrm{~min})$, the degradation levels were similar for all the materials, it was remarkable that after 120 min photolysis showed the lower degradation degree and the two composites without

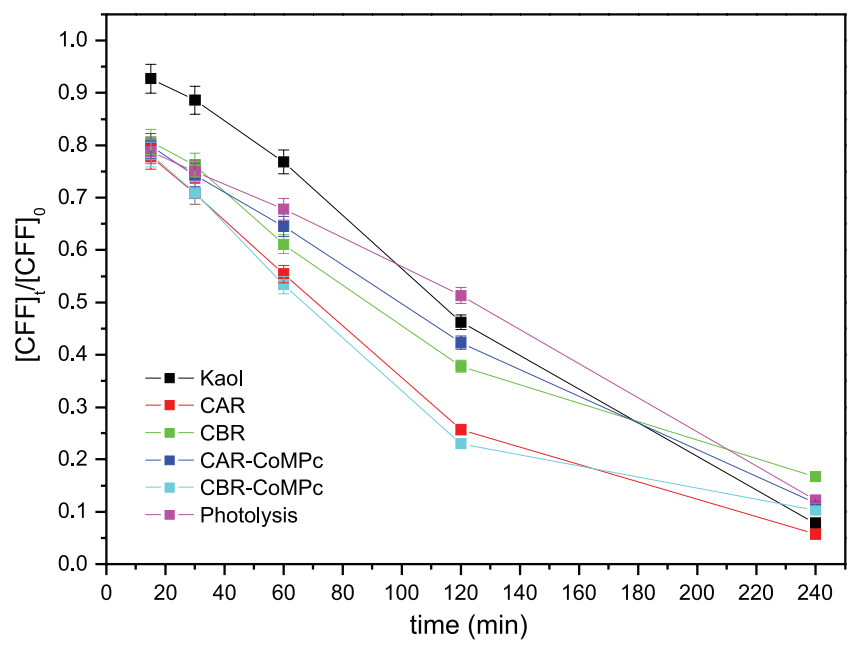

CoMPc showed a very high (ca. 80\%) degradation. Parent kaolinite again showed high activity, demonstrating that it was able to generate photoactive $\mathrm{OH} \bullet$ radicals.

The evolution of CFF under photocatalysis was also analyzed by mass spectrometry (Figure S8, SI section). From the $m / z$ values found and comparing with literature reports, ${ }^{48-50}$ its degradation route can be proposed (Figure 8). All the fragments detected by MS implied the opening of both the pyrimidine and imidazole rings, except the fragment with $\mathrm{m} / \mathrm{z} 242$ that involved only opening of the imidazole ring and oxidation of the terminal resulting groups. The easiness with which these rings can be opened seemed to be a key factor for degradation, and also for its determination by UV-Vis spectroscopy, as the resulting fragments may not absorb close to the absorption bands of CFF.

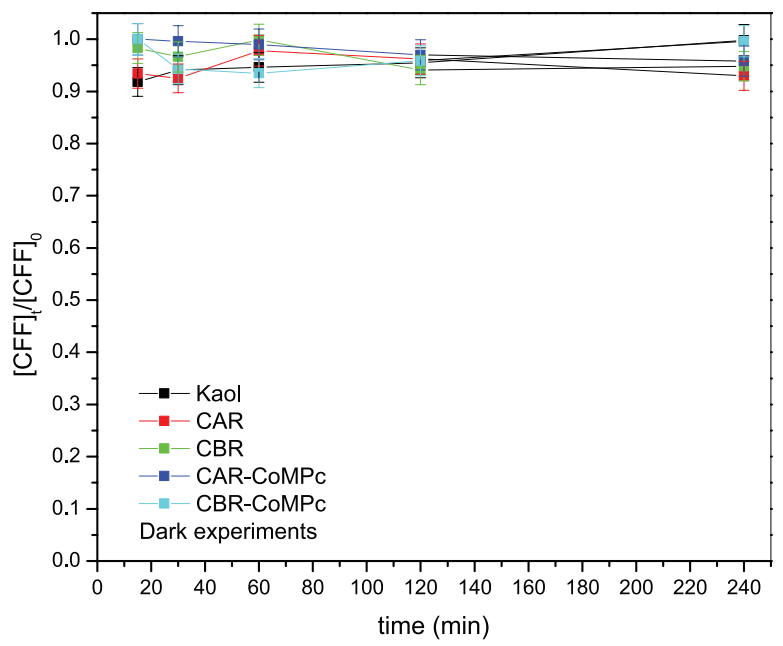

Figure 7. Kinetic behavior $\left(C_{t} / C_{0}\right)$ of the photodegradation of caffeine.

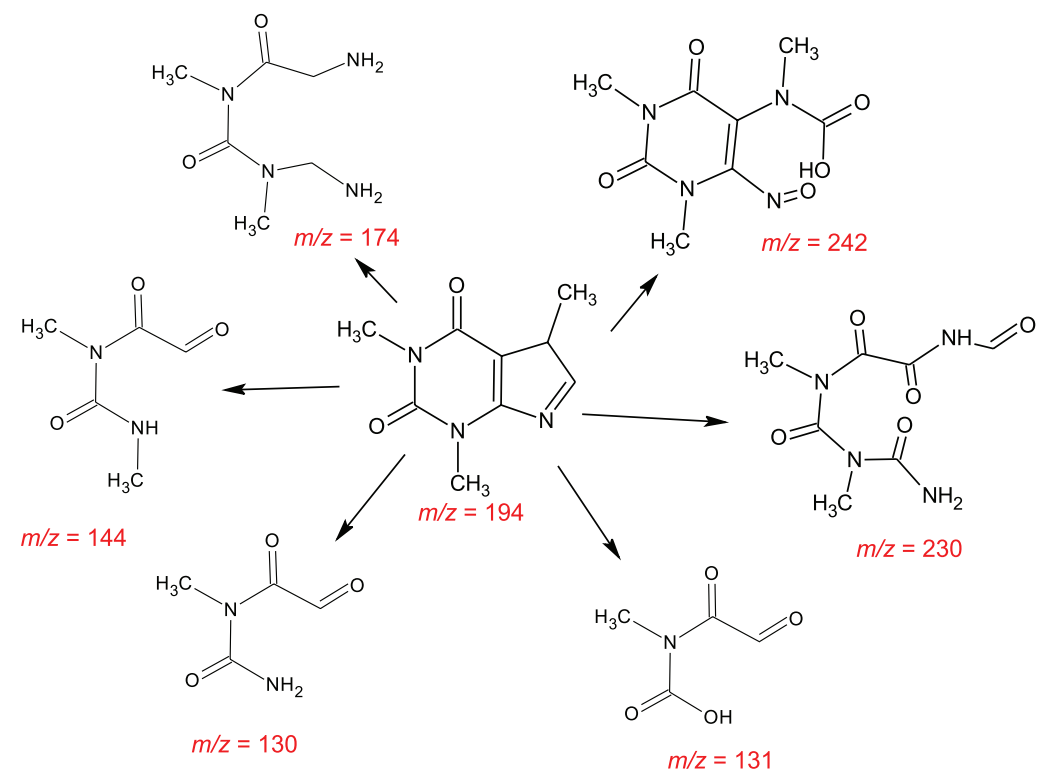

Figure 8. Proposal for the photocatalytic decomposition of CFF. 
Photodegradation of CFF has been previously reported by Marques et al. ${ }^{11}$ using composites based on acid activated carbon nanotubes and three different Ti-materials (one prepared by sol-gel and two other commercial ones from Evonik and Sigma-Aldrich), reaching 95\% degradation $\left(\lambda>350 \mathrm{~nm}\right.$; irradiance ca. $50 \mathrm{~mW} \mathrm{~cm}^{-2}$; mass of photocatalyst $1.0 \mathrm{~g} \mathrm{~L}^{-1}$, and time $180 \mathrm{~min}$ ). The best results were obtained for the composite formed by the carbon nanotubes and Sigma-Aldrich titania, a behavior which has been related to the larger size of the $\mathrm{TiO}_{2}$ crystallites and the better contact of these particles with carbon nanotubes.

According to Figures 3, 5 and 7, the solids containing $\mathrm{TiO}_{2}$-CoMPc promoted different degradation profiles reaching the higher efficiency for the substrate caffeine ( $90 \%$ of CFF, within $240 \mathrm{~min}$ ). Considering the data previously discussed, $\mathrm{TiO}_{2} / \mathrm{CoMPc}$ deposited on the kaolinite surface presented a much smaller particle size of semiconductor and a slightly larger surface area than the other materials (Table 2). However, it is important to remark that these were not key factors in the photocatalytic properties, since, despite these discrete differences, both had the same photocatalytic efficiency. In addition, the results obtained by FTIR before photocatalysis experiments (not shown) evidenced that both samples had residues of synthesis reagents adsorbed on their surface. These residues could compete with the active sites of the photocatalysts, impairing the photocatalysis efficiency. Thus, although photocatalytic efficiency was significant, it could have been even better if the surface of the particles were free of residues of synthesis and organic matter. This effect was confirmed by the reuse experiments, which demonstrated a decrease in the photocatalytic activity during the reuse of the solids.

When the degradation of the EOPs was monitored in relation to time, it was possible to calculate the reaction constant and the half-life time of the processes under study (Figure S9, SI section). Equation 2 relates the degradation time to the EOPs concentration:

$\ln \left(\frac{\mathrm{C}}{\mathrm{C}_{0}}\right)=\mathrm{k}^{\prime} \times \mathrm{t}$

where $\mathrm{k}^{\prime}=\mathrm{k}[\mathrm{SA}], \mathrm{k}$ is the rate constant of the reaction, [SA] is the concentration of active sites on the catalyst surface, $\mathrm{t}$ is the irradiation time: and $\mathrm{Co}$ and $\mathrm{C}$ represent the concentration of each EOP at the beginning of the reaction and at each time.

The formation of radicals responsible for dye degradation was correlated to high rate constants of the reaction ( $\left.\mathrm{k}^{\prime}\right)$ and low half-life times. However, for the constant k' to be high, which will influence the kinetics of degradation, the concentration of available active sites must also be high, since they are directly proportional to each other (equation 2).

Equation 3 allowed to calculate the time required to reduce to the half the concentration of organic compounds:

$\mathrm{t}^{\frac{1}{2}}=\ln \frac{2}{\mathrm{k}^{\prime}}$

where $\mathrm{t}^{1 / 2}$ is the half-life time.

According to equation 2, on plotting $-\ln \left(\mathrm{C} / \mathrm{C}_{0}\right)$ vs. $\mathrm{t}$ will lead to a straight line which slope is the rate constant of the reaction, $\mathrm{k}^{\prime}$. In the graphical representation of $-\ln \left(C / C_{0}\right)$ vs. $t$ (Figures 3, 5 and 7), a first order kinetics can be identified for all cases, indicating that all degradations followed the same mechanism.

Table 3 contains the values of k' obtained from Figures 3, 5 and 7, and their respective half-life times. The reaction presented values of $\mathrm{k}^{\prime}$ and $\mathrm{t}_{1 / 2}$ quite close for $\mathrm{TiO}_{2}$-CoMPc solids. On the other hand, the values of $\mathrm{k}$ ' and $t_{1 / 2}$ for the different substrates were relatively different, a given solid showing higher $\mathrm{k}$ ' and consequently smaller $\mathrm{t}_{1 / 2}$ for smaller and simple substrate molecules such as $\mathrm{CFF}$, and therefore lower photodegradation rate and much higher $\mathrm{t}^{1 / 2}$ for complex molecules such as PMT and TMP. $\mathrm{TiO}_{2}$-Co-MPc and $\mathrm{TiO}_{2}$ deposited on the kaolinite surface using acidic or alkaline routes were therefore equally efficient in the degradation of the different EOPs under study (evidenced by the caffeine study).

Literature studies for degradation of TMP, ${ }^{46} \mathrm{PMT}^{15}$ and $\mathrm{CFF}^{11}$ have reported that photodegradation was improved by the addition of oxidizing agents or by previous treatments for generation of acid active sites on the materials (Table 4). In our case, the reaction was carried out in water, without addition of oxidants, and although the degradation level reached was lower than in other studies, all the materials showed good capacity to generate the $\mathrm{OH} \bullet$ radicals needed for degradation of the resistant molecules considered.

\section{Evaluation of photodegradation mechanism}

Zhang and co-workers ${ }^{51-53}$ have shown the inhibiting effect of inorganic ions, such as chloride, on the photocatalytic degradation of organic pollutants. Most of them have shown that chloride ions existing in the solutions as salts or as degradation by-products, can promote the inhibition of the efficiency during a photocatalytic process. Thus, in order to evaluate the impact of the chloride ions, experiments were conducted with PMT using $16.6 \mathrm{mg} \mathrm{L}^{-1}$ of 
Table 3. First-order kinetic values of $\mathrm{k}^{\prime}, \mathrm{R}^{2}$, and $\mathrm{t}_{1 / 2}$ of the various photocatalysis experiments

\begin{tabular}{|c|c|c|c|c|c|c|c|c|c|}
\hline \multirow{3}{*}{ Sample } & \multicolumn{9}{|c|}{ First-order kinetics parameters } \\
\hline & \multicolumn{3}{|c|}{ Trimetoprim (TMP) } & \multicolumn{3}{|c|}{ Prometryn (PMT) } & \multicolumn{3}{|c|}{ Caffeine (CFF) } \\
\hline & k' & $\mathrm{R}^{2}$ & $\mathrm{t}^{1 / 2} / \min$ & $\mathrm{k}^{\prime}$ & $\mathrm{R}^{2}$ & $\mathrm{t}^{1 / 2} / \min$ & k' & $\mathrm{R}^{2}$ & $\mathrm{t}^{1 / 2} / \min$ \\
\hline Kaol & 0.005 & 0.99 & 28 & 0.001 & 0.99 & 48 & 0.011 & 0.95 & 20 \\
\hline CAR & 0.004 & 0.98 & 30 & 0.001 & 0.99 & 48 & 0.012 & 0.99 & 19 \\
\hline CBR & 0.001 & 0.95 & 48 & 0.0003 & 0.90 & 66 & 0.007 & 0.99 & 25 \\
\hline CAR-CoMPc & 0.003 & 0.98 & 34 & 0.001 & 0.98 & 48 & 0.009 & 0.97 & 22 \\
\hline CBR-CoMPc & 0.001 & 0.68 & 48 & 0.0004 & 0.97 & 61 & 0.009 & 0.97 & 22 \\
\hline Photolysis & 0.005 & 0.99 & 28 & 0.002 & 0.99 & 39 & 0.008 & 0.92 & 23 \\
\hline
\end{tabular}

CAR: composite acid route; CBR: composite basic route; CoMPc: cobalt(II) tetracarboxyphthalocyanine; $\mathrm{R}^{2}$ : regression coefficient; $\mathrm{k}^{\prime}$ : rate constants of the reaction; $\mathrm{t}^{1 / 2}$ : half-life time.

Table 4. Comparison of the results obtained in the present work with some literature results for degradation of TMP, PMT and CFF

\begin{tabular}{|c|c|c|c|c|c|}
\hline \multirow{2}{*}{ Catalyst } & \multicolumn{3}{|c|}{ Degradation / \% } & \multirow{2}{*}{ Oxidant } & \multirow{2}{*}{ Reference } \\
\hline & TMP & PMT & $\mathrm{CFF}$ & & \\
\hline Kaol & 70 & 38 & 92 & & this work \\
\hline CAR & 61 & 30 & 94 & & this work \\
\hline CBR & 28 & 11 & 83 & & this work \\
\hline CAR-CoMPc & 54 & 30 & 88 & & this work \\
\hline CBR-CoMPc & 33 & 11 & 90 & & this work \\
\hline $\mathrm{TiO}_{2}$ & 90 & & & $\mathrm{H}_{2} \mathrm{O}_{2}$ & 46 \\
\hline Degussa P-25 and UV-100 Hombikat & & 70 & & $\mathrm{H}_{2} \mathrm{O}_{2}$ and $\mathrm{K}_{2} \mathrm{~S}_{2} \mathrm{O}_{8}$ & 15 \\
\hline $\mathrm{TiO}_{2} /$ carbon nanotubes & & & 95 & & 11 \\
\hline
\end{tabular}

CAR: composite acid route; CBR: composite basic route; CoMPc: cobalt(II) tetracarboxymetallophthalocyanine; TMP: trimethoprim; PMT: prometryn; CFF: caffeine.

$\mathrm{NaCl}$ in the initial PMT solution. Figure 9 shows the evident inhibition with chloride ions; only around $2.5 \%$ of PMT was degraded in the presence of $\mathrm{NaCl}$, compared to the maximum of $38 \%$ of PMT degradation in the experiments without $\mathrm{NaCl} .^{51-54}$

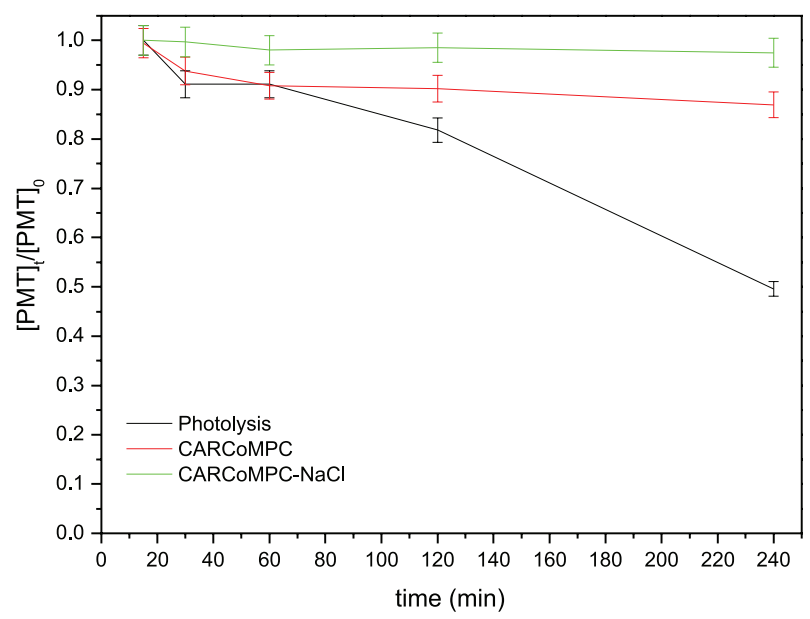

Figure 9. Photocatalytic degradation of PMT in the presence of $\mathrm{NaCl}$.
Based on these results, it is confirmed that when a certain concentration of $\mathrm{NaCl}$ existed in the reaction system, the degradation of PMT could not be initiated further. Moreover, results in Figure 10 showed the inhibiting effect of chloride ions on the constant rates ( $\mathrm{k}^{\prime}$ ) and half-life time $\left(\mathrm{t}^{1 / 2}\right)$ during PMT degradation. PMT degradation constant decreased from 0.003 on photolysis to $4.7 \times 10^{-4} \mathrm{mg} \mathrm{L}^{-1} \mathrm{~s}^{-1}$ when CAR-CoMPc was used as a catalyst for PMT photodegradation and to $9.7 \times 10^{-5} \mathrm{mg} \mathrm{L}^{-1} \mathrm{~s}^{-1}$ when the same catalyst was used in the presence of $16.6 \mathrm{mg} \mathrm{L}^{-1}$ $\mathrm{NaCl}$. The kinetic reaction constants in the presence of chloride ions were approximately 5 times lower than for PMT degradation, while the half-life time was 34 min for photolysis, 59 min when using CAR-CoMPc as catalyst, and $85 \mathrm{~min}$ when using the same catalyst in the presence of $\mathrm{NaCl}$, in agreement with the evolution of the degradation constants.

Two possible mechanisms have been proposed for the inhibition caused by inorganic ions on the photocatalytic activity of systems containing $\mathrm{TiO}_{2}$. The first one is a competitive adsorption of inorganic ions on the pristine 
$\mathrm{TiO}_{2}$ active surface which blocks adsorption sites for the target molecules. The second one is a possible scavenging effect of the adsorbed inorganic ions which consume photogenerated holes and, therefore, decrease the efficiency of the photocatalytic process. Moreover, according to Zalazar et al. ${ }^{55}$ the direct attack of the photogenerated hole is the most significant oxidative step in the photocatalytic oxidation of EOPs, which confirms the great importance of the adsorption ability of the EOPs and the hole quantity for an efficient photocatalytic mineralization.

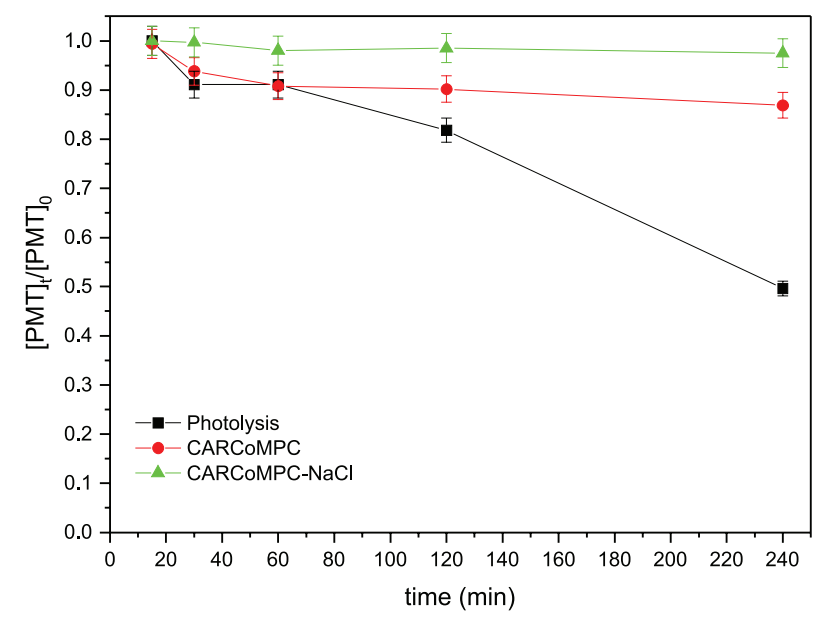

Figure 10. Kinetic behavior $\left(C_{l} / C_{0}\right)$ of the photodegradation of PMT with $\mathrm{NaCl}$.

\section{Conclusions}

Titanium dioxide and cobalt(II)-metallophthalocyanine were effectively immobilized on kaolinite surface, using an acid or a basic route for the hydrolysis of the Ti-isopropoxide precursor. The preparation treatments did not alter the basal spacing of kaolinite, but slightly modified the specific surface area and more significantly the band gap of the solid from $4.7 \mathrm{eV}$ in kaolinite based solids containing titanium dioxide to $1.78 \mathrm{eV}$ in solids containing cobalt(II)-metallophthalocyanine. In the presence of artificial light (from UV to visible), the solids showed moderate photocatalytic activity for removal of trimethoprim and prometryn (54 and 30\% total degradation rate), but much higher for caffeine ( $90 \%$ degradation rate) using solids with cobalt(II)-metallophthalocyanine. In all the photodegradation reactions the presence of by-products was confirmed by mass spectrometry, allowing to identify most of the fragments. In the cases of trimethoprim and prometryn, their rings were not broken, which strongly hindered their degradation, and it may alter the tracing of the process by UV-Vis spectroscopy, as the fragments containing these rings may absorb at the wavelengths used for the determination of the original molecules. In the case of caffeine, their two rings were easily opened, facilitating the degradation of the molecule and also the tracing of the process. The study by mass spectrometry corroborated the different degradation rates obtained by photocatalysis for the three studied molecules.

\section{Supplementary Information}

Supplementary information (molecular structures of organic pollutants, tables from XRD data, chemical reactions, thermal analysis, infrared spectra, $\mathrm{N}_{2}$ isotherm adsorption-desorption, UV-Vis absorption of nanocomposites solid state analysis, mass spectrometry) is available free of charge at http://jbcs.sbq.org.br as PDF file.

\section{Acknowledgments}

The authors thank a Cooperation Grant jointly financed by Universidad de Salamanca (Spain) and FAPESP (Brasil, 2016/50322-2). This study was financed in part by the Coordenação de Aperfeiçoamento de Pessoal de Nível Superior - Brasil (CAPES) - Finance Code 001. The Brazilian group acknowledges the support from the Brazilian research funding agencies Fundação de Amparo à Pesquisa do Estado de São Paulo (FAPESP) (2017/15482-1, 2016/01501-1), CAPES (317/15 and 0274-16) and Conselho Nacional de Desenvolvimento Científico e Tecnológico (CNPq) (305398/2015-6, 302668/2017-9, 311767/2015-0).

\section{References}

1. Grazieli, C.; Collins, C. H.; Quim. Nova 2011, 34, 665.

2. Michael, I.; Frontistis, Z.; Fatta-Kassinos, D. In Analysis, Removal, Effects and Risk of Pharmaceuticals in the Water Cycle: Occurrence and Transformation in the Environment (Comprehensive Analytical Chemistry), vol. 62; Petrovic, M., Barcelo, D., Pérez, S., eds.; Elsevier: Amsterdam, 2013, p. 345-407.

3. Bethi, B.; Sonawane, S. H.; Bhanvase, B. A.; Gumfekar, S. P.; Chem. Eng. Proc. Process. Int. 2016, 109, 178.

4. Geissen, V.; Mol, H.; Klumpp, E.; Umlauf, G.; Nadal, M.; van der Ploeg, M.; van de Zee, S. E. A. T. M.; Ritsema, C. J.; Int. Soil Water Conserv. Res. 2015, 3, 57.

5. Norman Network of Reference Laboratories, Research Centres and Related Organisations for Monitoring of Emerging Environmental Substances, available at http://www.normannetwork.net accessed in July 2019.

6. Luo, X.; Zheng, Z.; Greaves, J.; Cooper, W. J.; Song, W.; Water Res. 2012, 46, 1327.

7. Zhang, Y.; Wang, A.; Tian, X.; Wen, Z.; Lv, H.; Li, D.; Li, J.; J. Hazard. Mater. 2016, 318, 319. 
8. Oros-Ruiz, S.; Zanella, R.; Prado, B.; J. Hazard. Mater. 2013, 263, 28.

9. Ji, Y.; Xie, W.; Fan, Y.; Shi, Y.; Kong, D.; Lu, J.; Chem. Eng. J. 2016, 286, 16.

10. Liu, L.; Wan, Q.; Xu, X.; Duan, S.; Yang, C.; Food Chem. 2017, $219,7$.

11. Marques, R. R. N.; Sampaio, M. J.; Carrapiço, P. M.; Silva, C. G.; Morales-Torres, S.; Dražić, G.; Faria, J. L.; Silva, A. M. T.; Catal. Today 2013, 209, 108.

12. Marçal, L.; de Faria, E. H.; Nassar, E. J.; Trujillano, R.; Martín, N.; Vicente, M. A.; Rives, V.; Gil, A.; Korili, S. A.; Ciuffi, K. J.; ACS Appl. Mater. Interfaces 2015, 7, 10853.

13. Claver, A.; Ormad, P.; Rodríguez, L.; Ovelleiro, J. L.; Chemosphere 2006, 64, 1437.

14. Liu, J.; Hua, R.; Lv, P.; Tang, J.; Wang, Y.; Cao, H.; Wu, X.; Li, Q. X.; Sci. Total Environ. 2017, 579, 115.

15. Evgenidou, E.; Bizani, E.; Christophoridis, C.; Fytianos, K.; Chemosphere 2007, 68, 1877.

16. Araújo, F. R.; Baptista, J. G.; Marçal, L.; Ciuffi, K. J.; Nassar, E. J.; Calefi, P. S.; Vicente, M. A.; Trujillano, R.; Rives, V.; Gil, A.; Korili, S.; de Faria, E. H.; Catal. Today 2014, 227, 105.

17. Barbosa, L. V.; Marçal, L.; Nassar, E. J.; Calefi, P. S.; Vicente, M. A.; Trujillano, R.; Rives, V.; Gil, A.; Korili, S. A.; Ciuffi, K. J.; de Faria, E. H.; Catal. Today 2015, 246, 133.

18. Christoforidis, K. C.; Montini, T.; Bontempi, E.; Zafeiratos, S.; Jaén, J. J. D.; Fornasiero, P.; Appl. Catal., B. 2016, 187, 171.

19. Alharbi, S. K.; Kang, J.; Nghiem, L. D.; van de Merwe, J. P.; Leusch, F. D. L.; Price, W.E.; Process Saf. Environ. Prot. 2017, $2,1$.

20. Patil, P. N.; Bote, S. D.; Gogate, P. R.; Ultrason. Sonochem. 2014, 21, 1770.

21. Ratnasamy, P.; Srinivas, D.; Knözinger, H.; Adv. Catal. 2004, $48,1$.

22. Papoulis, D.; Komarneni, S.; Panagiotaras, D.; Nikolopoulou, A.; Li, H.; Yin, S.; Tsugio, S.; Katsuki, H.; Appl. Clay Sci. 2013, 83-84, 191.

23. Deng, F.; Zhao, X.; Pei, X.; Luo, X.; Li, W.; Au, C.; Sci. Adv. Mater. 2016, 8, 1079.

24. Dobrowolska, M.; Tivakornsasithorn, K.; Liu, X.; Furdyna, J. K.; Berciu, M.; Yu, K. M.; Walukiewicz, W.; Nat. Mater. 2012, 11, 444.

25. Mesgari, Z.; Gharagozlou, M.; Khosravi, A.; Gharanjig, K.; Spectrochim. Acta, Part A 2012, 92, 148.

26. Papoulis, D.; Komarneni, S.; Nikolopoulou, A.; Tsolis-Katagas, P.; Panagiotaras, D.; Kacandes, H. G.; Zhang, P.; Yin, S.; Sato, T.; Katsuki, H.; Nikolopoulou, A.; Li, H.; Yin, S.; Appl. Clay Sci. 2010, 50, 118.

27. Karamanis, D.; Ökte, A. N.; Vardoulakis, E.; Vaimakis, T.; Appl. Clay Sci. 2011, 53, 181.

28. de Faria, E. H.; Ricci, G. P.; Marçal, L.; Nassar, E. J.; Vicente, M. A.; Trujillano, R.; Gil, A.; Korili, S. A.; Ciuffi, K. J.; Calefi, P. S.; Catal. Today 2012, 187, 135.
29. Janíková, B.; Tokarský, J.; Mamulová Kutláková, K.; Kormunda, M.; Neuwirthová, L.; Appl. Clay Sci. 2017, 143, 345.

30. Kadish, K.; Smith, K. M.; Guilard, R. In The Porphyrin Handbook, vol. 11; Academic Press: Amsterdam, 1999.

31. Kimura, M.; Kuroda, T.; Ohta, K.; Hanabusa, K.; Shirai, H.; Kobayashi, N.; Langmuir 2003, 19, 4825.

32. da Silva, T. H.; de Souza, T. F. M.; Ribeiro, A. O.; Calefi, P. S.; Ciuffi, K. J.; Nassar, E. J.; Molina, E. F.; Hamer, P.; de Faria, E. H.; Dyes Pigm. 2016, 134, 41.

33. de Faria, E. H.; Lima, O. J.; Ciuffi, K. J.; Nassar, E. J.; Vicente, M. A.; Trujillano, R.; Calefi, P. S.; J. Colloid Interface Sci. 2009, $335,210$.

34. de Faria, E. H.; Ciuffi, K. J.; Nassar, E. J.; Vicente, M. A.; Trujillano, R.; Calefi, P. S.; Appl. Clay Sci. 2010, 48, 516.

35. Brinker, C. J.; Scherer, G. W.; Sol-Gel Science: The Physics and Chemistry of Sol-Gel Processing; Academic Press: Amsterdam, 1990.

36. Zbik, M. S.; Raftery, N. A.; Smart, R. S. C.; Frost, R. L.; Appl. Clay Sci. 2010, 50, 299.

37. Letaief, S.; Leclercq, J.; Liu, Y.; Detellier, C.; Langmuir 2011 , $27,15248$.

38. Dedzo, G. K.; Detellier, C.; Appl. Clay Sci. 2014, 97-98, 153.

39. da Silva, A. C.; Ciuffi, K. J.; dos Reis, M. J.; Calefi, P. S.; de Faria, E. H.; Appl. Clay Sci. 2016, 126, 251.

40. da Silva, T. H.; de Souza, T. F. M.; Ribeiro, A. O.; Ciuffi, K. J.; Nassar, E. J.; Silva, M. L. A.; de Faria, E. H.; Calefi, P. S.; Dyes Pigm. 2014, 100, 17.

41. Cintra, T. E.; Saltarelli, M.; Salmazo, R. M. D. F.; Silva, T. H.; Nassar, E. J.; Trujillano, R.; Rives, V.; Vicente, M. A.; de Faria, E. H.; Ciuffi, K. J.; Appl. Clay Sci. 2019, 168, 469.

42. Thommes, M.; Kaneko, K.; Neimark, A. V.; Olivier, J. P.; Rodriguez-Reinoso, F.; Rouquerol, J.; Sing, K. S. W.; Pure Appl. Chem. 2015, 87, 1051.

43. Tauc, J.; Mater. Res. Bull. 1970, 5, 721.

44. Pirbazari, A. E.; Procedia Mater. Sci. 2015, 11, 622.

45. González, B.; Trujillano, R.; Vicente, M. A.; Rives, V.; de Faria, E. H.; Ciuffi, K. J.; Korili, S. A.; Gil, A.; Environ. Chem. 2017, 14, 267.

46. Cai, Q.; Hu, J.; J. Hazard. Mater. 2017, 323, 527.

47. Kiss, A.; Rapi, S.; Csutorás, C.; Microchem. J. 2007, 85, 13.

48. Indermuhle, C.; Martín de Vidales, M. J.; Sáez, C.; Robles, J.; Cañizares, P.; García-Reyes, J. F.; Molina-Díaz, A.; Comninellis, C.; Rodrigo, M. A.; Chemosphere 2013, 93, 1720.

49. Gracia-Lor, E.; Rousis, N. I.; Zuccato, E.; Bade, R.; Baz-Lomba, J. A.; Castrignanò, E.; Causanilles, A.; Hernández, F.; KasprzykHordern, B.; Kinyua, J.; McCall, A. K.; van Nuijs, A. L. N.; Plósz, B. G.; Ramin, P.; Ryu, Y.; Santos, M. M.; Thomas, K.; de Voogt, P.; Yang, Z.; Castiglioni, S.; Sci. Total Environ. 2017, 609, 1582.

50. Lin, K. Y. A.; Lai, H. K.; Tong, S.; J. Colloid Interface Sci. 2018, 514, 272. 
51. Ma, Y.; Zhang, G.; Chem. Eng. J. 2016, 288, 70.

52. Fida, H.; Guo, S.; Zhang, G.; J. Colloid Interface Sci. 2015, 442, 30.

53. Deng, L.; Xie, Y.; Zhang, G.; Chin. J. Catal. 2017, 38, 379.

54. Bahnemann, D. W.; Mehle, A.; Dražić, G.; Štrancar, J.; Dillert, R.; Krivec, M.; Phys. Chem. Chem. Phys. 2014, 16, 14867.
55. Zalazar, C. S.; Martin, C. A.; Cassano, A. E.; Chem. Eng. Sci. 2005, 60, 4311.

Submitted: January 28, 2019

Published online: July 31, 2019 